\title{
ALL THE INFINITE NEST ALGEBRAS ARE ISOMORPHIC
}

\author{
ALVARO ARIAS
}

(Communicated by Paul S. Muhly)

\begin{abstract}
All the infinite nest algebras in a separable Hilbert space are completely isomorphic.
\end{abstract}

In this note we prove that all the infinite nest algebras in a separable Hilbert space are completely isomorphic to each other. The simplest candidate resembles one of the isomorphic representations of $H^{\infty}$ given by Wojtaszczyk [W]. He proved that $H^{\infty}$ is isomorphic to $\left(\sum_{i=1}^{n} \bigoplus A_{n}\right)_{\infty}$ where $A_{n}=\operatorname{span}\left\{z^{i}\right\}_{i=0}^{n} \subset$ $A$ and $A$ is the disc algebra.

For this paper $H$ denotes a separable Hilbert space. A nest of projections in $H$ is any linearly ordered set $\mathscr{P}$ of orthogonal projections closed in the strong operator topology and containing 0 and $I$. The nest algebra induced by $\mathscr{P}$ is the set of all operators $T$ in $B(H)$ that leaves invariant every element of $\mathscr{P}$; i.e.,

$$
\operatorname{Alg} \mathscr{P}=\{T \in B(H):(I-P) T P=0 \text { for every } P \in \mathscr{P}\} .
$$

Two of the most common nests of projections are the following:

The discrete nest. Let $\left(e_{i}\right)$ be an orthonormal basis for $H$, and for $0 \leq n \leq$ $\infty$ let $P_{n}$ be the orthogonal projections onto $\overline{\operatorname{span}}\left\{e_{i}: 1 \leq i \leq n\right\}$.

The Volterra nest. Let $H=L_{2}[0,1]$, and for $0 \leq t \leq 1$ let $P_{t}$ be the orthogonal projection onto the subspace of all functions supported on $[0, t]$.

These examples are fundamental and have motivated a big part of the theory. If $\mathscr{P}$ is the discrete nest, $\mathrm{Alg} \mathscr{P}$ is the set of all "upper triangular" operators with respect to the orthonormal basis; and the Volterra nest is the canonical example of a continuous nest (without gaps). Indeed, Larson [L] proved that any two continuous nests are similar; i.e., If $\mathscr{P}$ and $\widetilde{P}$ are continuous nests, then there exists $V \in B(H)$ invertible such that $\operatorname{Alg} \mathscr{P}=V^{-1} \mathrm{Alg} \widetilde{\mathscr{P}} V$. Such an isomorphism is called spatial.

We distinguish between algebraic isomorphism and Banach space isomorphism between nest algebras. The first one preserves the linear and the algebraic properties of the nest algebra.

Ringrose $[R]$ proved that every algebraic isomorphism between nest algebras is spatial; and it is well known that the discrete and the Volterra nest algebras

Received by the editors July 10, 1990 and, in revised form, November 16, 1990.

1980 Mathematics Subject Classification (1985 Revision). Primary 46L35; Secondary 47D25.

Key words and phrases. Nest algebra, completely isomorphic.

Supported in part by NSF DMS-87 03815. 
are not similar. Nevertheless, they cannot be distinguished by their Banach space structure.

Let $V, W \subset B(H)$ and $u: V \rightarrow W$ a linear map. Define

$$
u_{n}: M_{n}(V) \rightarrow M_{n}(W) \quad \text { by } u_{n}\left(\left[a_{i j}\right]\right)=\left[u\left(a_{i j}\right)\right] .
$$

Since $M_{n}(V), M_{n}(W) \subset M_{n}(B(H)) \equiv B\left(H^{n}\right)$, they have a natural norm induced by $B\left(H^{n}\right)$. We say that $u$ is completely bounded if $\|u\|_{c b}=\sup _{n}\left\|u_{n}\right\|<$ $\infty$. Moreover, if both $u$ and $u^{-1}$ are completely bounded, then $u$ is a complete isomorphism.

The main result is the following:

Theorem 1. Let $\operatorname{Alg} \mathscr{P}$ be an infinite nest algebra in a separable Hilbert space. Then Alg $\mathscr{P}$ is completely isomorphic to $\left(\sum_{n=1}^{\infty} \bigoplus \mathscr{T}_{n}\right)_{\infty}$, where $\mathscr{T}_{n}$ is the algebra of all upper triangular $n \times n$ matrices.

The proof of Theorem 1 follows from Pelczyński's decomposition method (see $[\mathrm{Pel}]$ ) and the semidiscreteness of the nest algebras proved by Paulsen, Power, and Ward [PPW].

Theorem 2 (Paulsen, Power, and Ward [PPW]). Let $\mathscr{A}=\operatorname{Alg} \mathscr{P}$ be a nest algebra acting on a separable Hilbert space $H$. Then there exist

(i) a sequence $\mathscr{A}_{n}$ of finite-dimensional nest algebras;

(ii) $\sigma$-weakly continuous completely contractive maps $\phi_{n}: \mathscr{A}_{\rightarrow} \rightarrow \mathscr{A}_{n}$;

(iii) $\sigma$-weakly continuous completely isometric homomorphisms $\psi_{n}: \mathscr{A}_{n} \rightarrow$ $\mathscr{A}$, such that $\psi_{n}\left(\phi_{n}(A)\right) \rightarrow A \quad \sigma$-weakly for all $A$ in $\mathscr{A}$.

Pełczyński's decomposition method says that if $X$ and $Y$ are Banach spaces and if $X$ embeds complementably in $Y, Y$ embeds complementably in $X$ and $X$ is isomorphic to its $l_{\infty}$-sum; i.e., $X \approx\left(\sum \bigoplus X\right)_{l_{\infty}}$, then $X$ is isomorphic to $Y$. Moreover, if the embeddings are completely bounded and completely complementable and the isomorphism is a complete isomorphism then $X$ and $Y$ are completely isomorphic.

It is clear that $\left(\sum_{n=1}^{\infty} \bigoplus \mathscr{T}_{n}\right)_{\infty}$ is completely isomorphic to its $l_{\infty}$-sum. Moreover, if $\mathscr{P}$ is infinite it is easy to see that $\left(\sum_{n=1}^{\infty} \bigoplus \mathscr{T}_{n}\right)_{\infty}$ embeds completely bounded and completely complemented in Alg $\mathscr{P}$. To see this, notice that we need only $n$ elements of $\mathscr{P}$ to embed $\mathscr{T}_{n}$ in $\mathrm{Alg} \mathscr{P}$. It remains to prove that Alg $\mathscr{P}$ embeds completely bounded and completely complemented in $\left(\sum_{n=1}^{\infty} \bigoplus \mathscr{T}_{n}\right)_{\infty}$.

We prove Theorem 1 first for multiplicity free nests. The advantage here is that the finite nest algebras $\mathscr{A}_{n}$ of Theorem 2 are the $\mathscr{T}_{n}$ 's. This is immediate from the proof of Theorem 2 .

Lemma 3. Let Alg $\mathscr{P}$ be an infinite multiplicity free nest algebra in a separable Hilbert space. Then $\mathrm{Alg} \mathscr{P}$ is completely isomorphic to $\left(\sum_{n=1}^{\infty} \bigoplus \mathscr{T}_{n}\right)_{\infty}$, where $\mathscr{T}_{n}$ is the algebra of all upper triangular $n \times n$ matrices.

Proof. Use Theorem 2 and the previous remark to find for every $n$, maps $\phi_{n}: \operatorname{Alg} \mathscr{P} \rightarrow \mathscr{T}_{n}$ and $\psi_{n}: \mathscr{T}_{n} \rightarrow$ Alg $\mathscr{P}$ with the required properties. Define $\Phi: \operatorname{Alg} \mathscr{P} \rightarrow\left(\sum_{n=1}^{\infty} \bigoplus \mathscr{T}_{n}\right)_{\infty}$ by

$$
\Phi(T)=\left(\phi_{n}(T)\right)_{n}
$$


By Theorem 2 (ii), (iii), one has that $\Phi$ is completely isometric. It remains to prove that the embedding is completely complemented. Let $\mathscr{U}$ be a free ultrafilter on the natural numbers and define $\Psi:\left(\sum_{n=1}^{\infty} \bigoplus \mathscr{T}_{n}\right)_{\infty} \rightarrow \operatorname{Alg} \mathscr{P}$ by

$$
\Psi\left(\left(T_{n}\right)\right)=\lim _{n \in \mathscr{U}} \psi_{n}\left(T_{n}\right),
$$

where the limit is taken in the $\sigma$-weak topology.

It is easy to see that $\Psi$ is well defined and is completely bounded. Theorem 2(iii) implies says that $\Psi \Phi=\mathrm{id}$. Then $\Phi \Psi$ gives the desired projection.

Proof of Theorem 1. Just as in the proof of Lemma 3, use Theorem 2 to show that $\mathrm{Alg} \mathscr{P}$ embeds in $\left(\sum \bigoplus \mathscr{A}_{n}\right)_{\infty}$ completely complemented for some finitedimensional nest algebras $\mathscr{A}_{n}$ 's.

Let $\widetilde{\mathscr{P}}$ be a continuous nest of multiplicity infinity. We clearly have that $\left(\sum \bigoplus \mathscr{A}_{n}\right)_{\infty}$ embeds in Alg $\widetilde{\mathscr{P}}$ completely complemented. The similarity Theorem (see [L]) tells us that $\mathrm{Alg} \widetilde{\mathscr{P}}$ is similar to a continuous nest of multiplicity one, and the latter is completely isomorphic to $\left(\sum \bigoplus \mathscr{T}_{n}\right)_{\infty}$. Therefore, $\operatorname{Alg} \mathscr{P}$ embeds completely bounded and completely complemented in $\left(\sum \bigoplus \mathscr{T}_{n}\right)_{\infty}$.

Remark. $\left(\sum \bigoplus \mathscr{T}_{n}\right)_{\infty}$ does not have the approximation property. Notice that $M_{n}$ 1-embeds into $\mathscr{T}_{2 n}$ (in the upper right corner); therefore, $\left(\sum \bigoplus M_{n}\right)_{\infty} 1$ embeds into $\left(\sum \bigoplus \mathscr{T}_{n}\right)_{\infty}$. Since $B(H)$ is isomorphic to $\left(\sum \bigoplus M_{n}\right)_{\infty}$ and does not have the approximation property (see [S]), the result follows.

Remark. $\left(\sum \bigoplus \mathscr{T}_{n}\right)_{\infty}$ is not isomorphic to $B(H)$. The result is analogous to the one that says that $H^{\infty}$ is not isomorphic to $L_{\infty}$, (see [Pe2]) but is deeper. We are indebted to G. Pisier for pointing it out to us. It follows from a result of his that says that $H^{\infty}$ is not isomorphic to a quotient of a $C^{*}$-algebra (see [DGT]); whereas $H^{\infty}$ is isomorphic to a quotient of $\mathrm{Alg} \mathscr{P}$, the discrete nest algebra (see [Po]).

The weaker statement $\left(\sum \bigoplus \mathscr{T}_{n}\right)_{\infty}$ is not completely isomorphic to $B(H)$, is easy because $\left(\sum \bigoplus \mathscr{T}_{n}\right)_{\infty}$ is not a $\lambda$-injective operator space for any $\lambda \geq 1$. If it were, then the main triangular projection on $M_{n}$ would be uniformly bounded.

\section{ACKNOWLEDGMENT}

The author wants to thank Professors G. Pisier and R. Smith for useful discussions.

\section{REFERENCES}

[DGT] W. Davis, D. Garling, and N. Tomczak-Jaegermann, The complex convexity of quasinormed linear spaces, J. Funct. Anal. 55 (1984), 110-150.

[L] D. Larson, Nest algebras and similarity transformations, Ann. of Math. 121 (1988), 409427.

[PPW] V. Paulsen, S. Power, and J. Ward, Semidiscreteness and dilations of nest algebras, J. Funct. Anal. 80 (1988), 76-87.

[Pe1] A. Petczyński, Projections in certain Banach spaces, Studia Math. 19 (1960), 209-228.

[Pe2] _ Banach spaces of analytic functions and absolutely summing operators, CBMS Regional Conf. Ser. 30, Amer. Math. Soc., Providence, R. I., 1977.

[Po] S. Power, Analysis in nest algebras, Surveys of Recent Results in Operator Theory (J. Conway, ed.), Pitman Research Notes in Math., Longman, vol. 192, 1988, pp. 189-234. 
[R] J. R. Ringrose, On some algebras of operators II, Proc. London Math. Soc. (3) 16 (1966), 385-402.

[S] A. Szankowski, $B(H)$ does not have the approximation property, Acta Math. 147 (1981), 89-108.

[W] P. Wojtaszczyk, On projections in spaces of bounded analytic functions, with applications, Studia Math. 65 (1979), 147-173.

Department of Mathematics, Texas A \& M University, College Station, Texas 77843

Current address: Department of Theoretical Mathematics, The Weizmann Institute of Science, Rehovot, Israel 